\title{
FICOPERIFÍTON ASOCIADO A MACRÓFITAS EN LA CIÉNAGA CERRO DE SAN ANTONIO, MAGDALENA-COLOMBIA
}

\author{
PHYCOPERIPHYTON ASSOCIATED TO MACROPHYTES IN THE SWAMP OF CERRO SAN \\ ANTONIO, MAGDALENA-COLOMBIA
}

Farid Jesús Osorio-Ávila y Gustavo Adolfo Manjarres-García

\begin{abstract}
RESUMEN
El estudio de algas del perifiton en humedales tropicales, permite interpretar la dinámica de estos ecosistemas con importancia ecológica y con vocación para ofrecer diferentes servicios a la población humana. El propósito de este trabajo fue determinar la estructura del ensamble ficoperifítico y evaluar su agremiación o preferencia de sustrato (macrófitas), en una ciénaga tropical. Se registraron 48 especies, los grupos de clorófitas y diatomeas, fueron dominantes. El ensamble algal mostró baja variación en cuanto a los índices comunitarios (diversidad, dominancia, riqueza), sin embargo se hallaron diferencias en las abundancias del ficoperifiton de cada una de las macrófitas evaluadas. También se encontraron diferencias en cuanto a la selectividad de sustrato de algunas especies tales como Closterium kuetzingii (Brébisson), Cocconeis placentula (Ehrenberg) y Hantzschia sp. (Ehrenberg), las cuales solo estuvieron presentes en las raíces de Eichhornia crassipes, a su vez Coelastrum sphaericum (Nägeli) solo se encontró en raíces de Pistia stratiotes. Se da el primer registro de la especie Ankistrodesmus fusiformis (Corda), para las ciénagas del departamento del Magdalena.
\end{abstract}

PALABRAS CLAVE: Agremiación, ensamble, ficoperifíton, humedales, macrófitas

\begin{abstract}
The study of periphyton algae in tropical wetlands to interpret the dynamics of these ecosystems with ecological importance and vocation to offer different services to the human population. The purpose of this study was to determine the structure of the assembly of periphyton and evaluate their aggregation or substrate preference (macrophytes) in a tropical swamp. We reported 48 species, they are presented as dominant group chlorophytic and diatoms, algal assemblage showed low variation in community indices (diversity, dominance, richness), however, the ficoperifiton abundance between macrophytes were found was similar. Moreover, differences in substrate selectivity of some species as Closterium kuetzingii (Brebisson), Cocconeis placentula (Ehrenberg), and Hantzschia sp. (Ehrenberg) which were only present in the roots of E. crassipes, in turn Coelastrum sphaericum (Nägeli) only found in roots of P. stratiotes. The first record of the species Ankistrodesmus fusiformis (Corda), to the swamps of the departamento del Magdalena.
\end{abstract}

KEY WORDS: Assembly, periphyton, macrophytes, unionization, wetlands

\section{INTRODUCIÓN}

El perifiton es una compleja comunidad de microorganismos tales como algas, bacterias, hongos y protozoos, adheridos a sustratos de cualquier tipo de material, natural o artificial, que se encuentre sumergido (Wetzel, 2001). El estudio de ficoperifíton en Colombia se ha desarrollado principalmente en embalses (Sierra 74 y Ramírez, 2000), lagos (Donato et al., 1996) y ríos (Hernández-Atilano et al., 2005; Montoya y Aguirre,
2007), con temáticas desde la ecología descriptiva, estructura de la microbiota, distribución espaciotemporal, colonización y sucesión de las especies en sustratos artificiales, sin embargo los estudios del perifiton en las ciénagas son escasos (Montoya-Moreno y Aguirre, 2009).

Las variables que se destacan por su importancia para la comunidad algal son, la velocidad del flujo, radiación solar, temperatura del agua (Wilby et al., 1998); 
también la herbivoría, la presencia de macrófitas en altas densidades y las condiciones de calidad del agua, son variables asociadas a la acumulación de perifiton en las macrófitas (Sand-Jensen, 1983). Sin embargo, las variables que afectan o regulan el crecimiento de las macrófitas y el perifiton, son específicos de cada ecosistema y presentan variación espacial y temporalmente (Flynn et al., 2002), por lo que se requiere analizar cada ecosistema de forma particular, ya que potencialmente ofrece nueva información específica de ese tipo de ambientes.

En Colombia, Montoya y Aguirre (2008, 2013) desarrollaron varias investigaciones en torno a la asociación entre microalgas y macrófitas, en el complejo cenagoso de Ayapel, en el departamento de Córdoba, dentro de los principales resultados encontraron que la comunidad ficoperifítica asociada a macrófitas, presenta índices comunitarios poco fluctuantes y sin diferencias en la estructura y composición de la comunidad entre las raíces de diferentes macrófitas.

El análisis de las relaciones entre la diversidad y el ambiente, es una importante herramienta para comprender los mecanismos de respuesta de las comunidades biológicas ante eventuales alteraciones en los ecosistemas (Rivera y Donato, 2008). Desde el punto de vista de conservación y calidad ambiental las microalgas perifíticas poseen atributos importantes para la bioindicación, ya que habitan numerosos ambientes acuáticos, presentan ciclos de vida corto y responden más rápidamente a los cambios ambientales que otros organismos (McCormick y Stevenson, 1998). Este estudio genera información de línea base para la caracterización de la comunidad de algas perifíticas asociadas a macrófitas en una ciénaga tropical.

\section{MATERIALES Y MÉTODOS}

\section{Área de estudio}

La ciénaga del Cerro de San Antonio, está ubicada en el departamento del Magdalena, entre los municipios Cerro de San Antonio y Concordia, contigua al corregimiento de Rosario de Chengue ( $10^{\circ} 15^{\prime} 52^{\prime \prime} \mathrm{N}$ y $\left.74^{\circ} 51^{\prime} 01^{\prime \prime} \mathrm{W}\right)$, a una altitud de $50 \mathrm{~m}$, hace parte del sistema de planos inundables de la cuenca baja del río Magdalena, al cual se conecta a través de un canal de aproximadamente 4,8 $\mathrm{km}$; el sector cuenta con pendientes menores al $7 \%$, con drenaje pobre, limita al oriente con una zona de lomeríos fluvio-gravitacionales con pendientes menores al $25 \%$ (IGAC, 2008), estas características hacen que sea clasificada como Ciénaga Tipo 2, de acuerdo con la descripción de Arias (1986).
El promedio anual de temperatura ambiental es de $28{ }^{\circ} \mathrm{C}$, la humedad relativa es del $60 \%$ y su régimen pluviométrico es de tipo bimodal, con precipitaciones promedio mensuales mínimas que van de 8 a $10 \mathrm{~mm}$ entre enero y febrero y máximas de $147 \mathrm{~mm}$ en mayo y $130 \mathrm{~mm}$ en octubre. La cuenca inmediata de la ciénaga se ve sometida periódicamente a eventos de inundación como resultado del aumento de caudales del río Magdalena y posterior desborde de aguas de la ciénaga (Barrios y Ortega, 1999).

Las estaciones de muestreo (Figura 1) se seleccionaron teniendo en cuenta la presencia de macrófitas en altas densidades así:

Estación 1. Caño Cerro de San Antonio (10¹7’12,5”N y $074^{\circ} 50^{\prime} 33,8$ ' W), en la desembocadura del caño hacia la ciénaga, se caracteriza por presentar un flujo de corriente continua, lo que genera que las macrófitas (flotantes y enraizadas) que entran a la ciénaga, provenientes del rio, se reagrupen en las orillas.

Estación 2. Pajarón $\left(10^{\circ} 16^{\prime} 07,7^{\prime} \mathrm{N}\right.$ y: 07451’12,2”W), este sector es más abierto con zonas de profundidad promedio de $52 \mathrm{~cm}$, las macrófitas se encuentran dispersas por acción del viento $(1,0 \mathrm{~m} / \mathrm{s})$.

Estación 3. Rosario de Chengue $\left(10^{\circ} 15^{\prime} 14,7^{\prime \prime} \mathrm{N}\right.$ y $\left.074^{\circ} 51^{\prime} 02,0^{\prime} \mathrm{W}\right)$, es una zona con profundidad moderada $(90 \mathrm{~cm})$, con abundancia de macrófitas en forma de parches que se asemejan a pequeñas islas móviles dentro del sistema.

\section{Fase de campo}

Los muestreos se realizaron durante el periodo de aguas altas entre los meses de julio y agosto. Mediante inspección visual se estudió la zona de muestreo para determinar las especies de macrófitas más abundantes. Se recolectó una fracción de la parte sumergida de las raíces de las especies de macrófitas dominantes: Eichhornia crassipes (Mart), Pistia stratiotes (Linneaus) y Nelumbo lutea (Wild), sin embargo, dado que no todas las macrófitas estuvieron presentes en las tres estaciones de muestreo, se recolectaron muestras integrales de $5 \mathrm{~cm}^{2}$ de las raíces de tres individuos de las especies seleccionadas. Las raíces se cortaron con la ayuda de pequeñas tijeras podadoras, posteriormente fueron depositadas en frascos de plástico de $50 \mathrm{ml}$ y preservadas en solución Transeau (Bicudo y Menezes, 2006). 


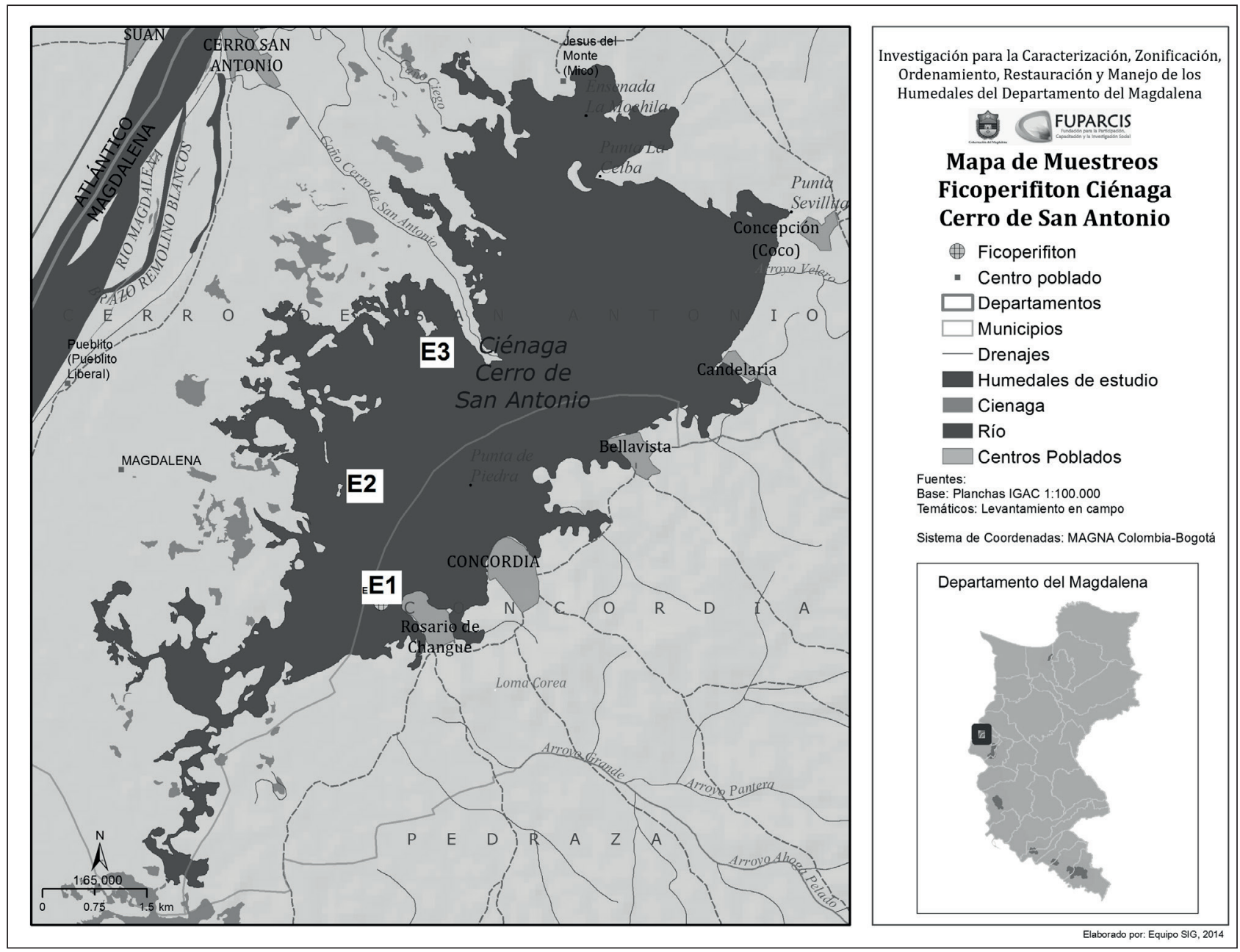

Figura 1. Mapa de la ciénaga Cerro de San Antonio. Estaciones de muestreo; de Sur a Norte: E1 (Rosario de Chengue), E2 (Pajaron) y E3 (Caño Cerro de San Antonio). Elaboró grupo de SIG FUPARCIS.

\section{Fase de laboratorio}

Las muestras recolectadas se llevaron al laboratorio, las raíces se rasparon con ayuda de un cepillo dental con lo cual se separó el material perifítico de los restos vegetales. Las muestras fueron identificadas en microscopia óptica con microscopio Nikon Eclipse 200, con cámara integrada, se utilizó un objetivo ocular de 100X; para las diatomeas el material que presentó abundante materia orgánica se limpió mediante el método de oxidación con peróxido de hidrógeno (CEN/ TC 230, 2002).

Para hacer la determinación de las especies ficoperifíticas se contó con la siguiente bibliografía: para géneros de microalgas Bourrelly (1970, 1972 y 1981), para Cyanophyceae Komárek y Anagnostidis $(2005,1999)$; para Bacillariophyceae Lange-Bertalot (2001), Krammer y Lange-Bertalot $(1986,1988,1991)$. Además se utilizaron bases de datos digitales (Guiry, 2015).

Para el análisis cuantitativo, las muestras se concentraron por sifoneo hasta un volumen de $10 \mathrm{ml}$. El conteo se realizó bajo un microscopio Nikon Eclipse 200, en cámara de conteo Sedgwick-Rafter de $1 \mathrm{ml}$ y se procedió a contar campos aleatorios hasta 100 células del taxón más abundante (Ramírez, 2000), la cuantificación de los individuos se obtuvo mediante la fórmula propuesta por Hauer y Lamberti (2007), donde la densidad se expresa en número de individuos por área de superficie $\left(\mathrm{cm}^{2}\right)$.

\section{Análisis de datos}

La estructura de la comunidad se evaluó mediante índices ecológicos; riqueza de especies, diversidad (Shannon) y dominancia (Simpson). A demás se realizó una revisión 
bibliográfica para algunas de las especies registradas de interés por su uso potencial en bioindicación. Se realizaron gráficas de barras en Excel, para representar las abundancias absolutas de algas perifíticas de las diferentes macrófitas estudiadas. Para evaluar la relación entre el ficoperifiton y las especies de macrófitas se hizo un análisis de componentes principales (ACP), con una matriz de correlación de las abundancias de las especies ficoperifíticas encontradas en cada sustrato. Se realizó un análisis de agrupamiento (Cluster), mediante un índice de disimilitud, para separar subgrupos de las macrófitas evaluadas con relación a las densidades ficoperifíticas. El paquete estadístico utilizado para los diferentes análisis estadísticos fue el PAST ver. 2.17c.

\section{RESULTADOS}

\section{Composición y agremiación ficoperifítica}

Se contaron 917 células, distribuidas en 32 géneros y 48 especies, las clases Bacillariophyceae y Chlorophyceae con 20 y 19 especies respectivamente, fueron las que presentaron la mayor riqueza, seguidas de Cyanophyceae con cinco y Fragillariophyceae con tres especies. De la familia Scenedesmaceae (Chlorophyceae), se registró la mayor densidad con 1600 células $/ \mathrm{cm}^{2}$, seguido de Gomphonemataceae (Bacillariophyceae) con 75 células $/ \mathrm{cm}^{2}$. La familia Cocconeidaceae (Bacillariophyceae) fue la menos abundante con 2 células/ $\mathrm{cm}^{2}$ (Figura 2).

La macrófita E. crassipes, presentó el mayor número de células asociadas a sus raíces con 409, seguida de Pistia stratiotes con 222 y Nelumbo lutea con 214 células. Algunas especies ficoperifíticas fueron exclusivas para algún sustrato (macrófitas). Así, Closterium küetzingii, Cocconeis placentula, Desmodesmus denticulatos, Hantzschia sp., Pediastrum tetras y Scenedesmus sp. fueron exclusivas de las raíces de E. crassipes y la especie Coelastrum sphaericum de las raíces de P. stratiotes (Figura 3).

En cuanto a la dominancia de ficoperifiton en cada macrófita, la especie dominante en raíces de $E$. crassipes fue Anabaena sp. con 45 células, por otra parte Tetraëdrum mínimum dominó en N. lutea con 15 células y Crucugenia fenestrata fue abundante en raíces de Pistia stratiotes con 19 células (Figura 3).

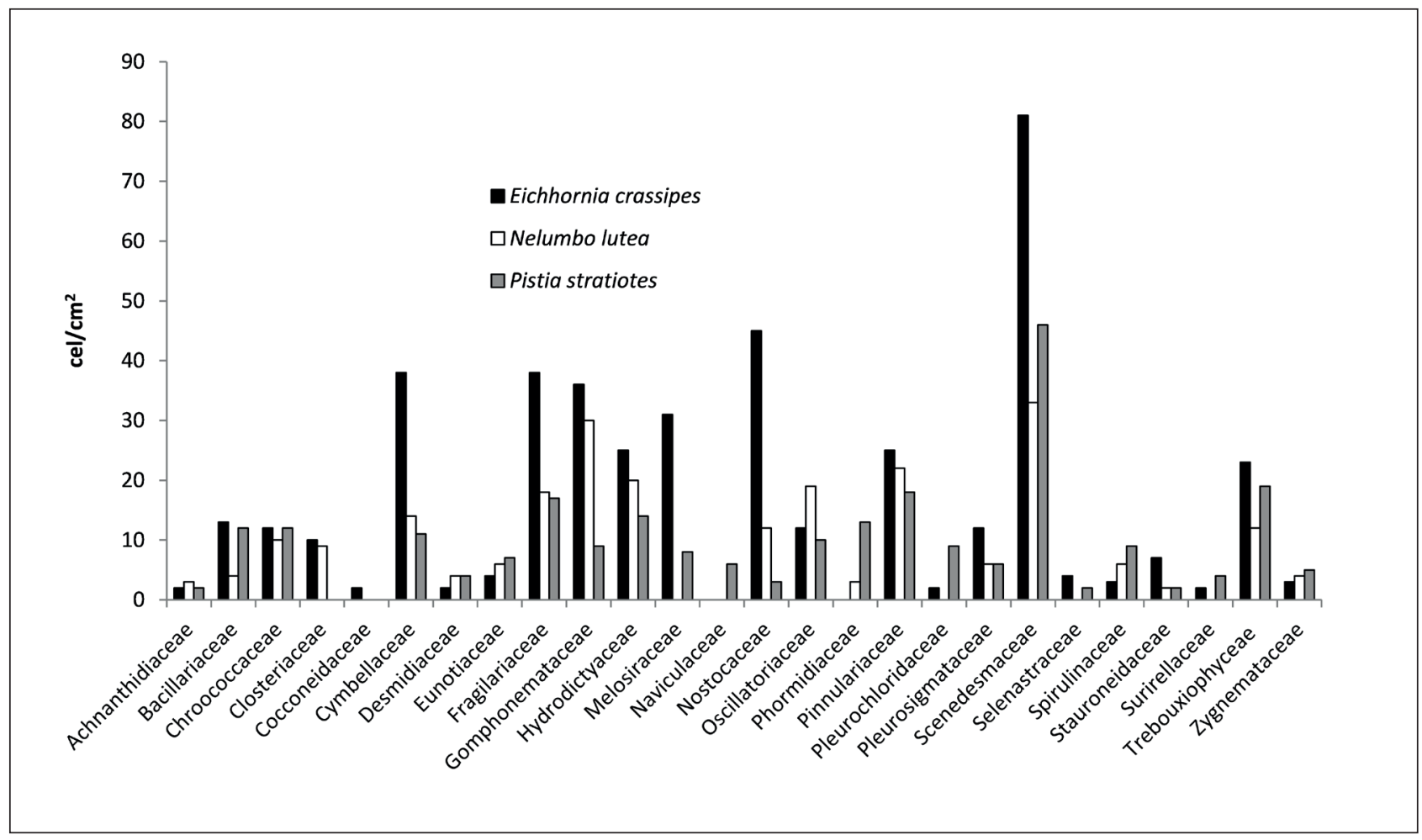

Figura 2. Abundancia relativa de familias ficoperifíticas asociadas a raíces de macrófitas en la Ciénaga Cerro de San Antonio. 


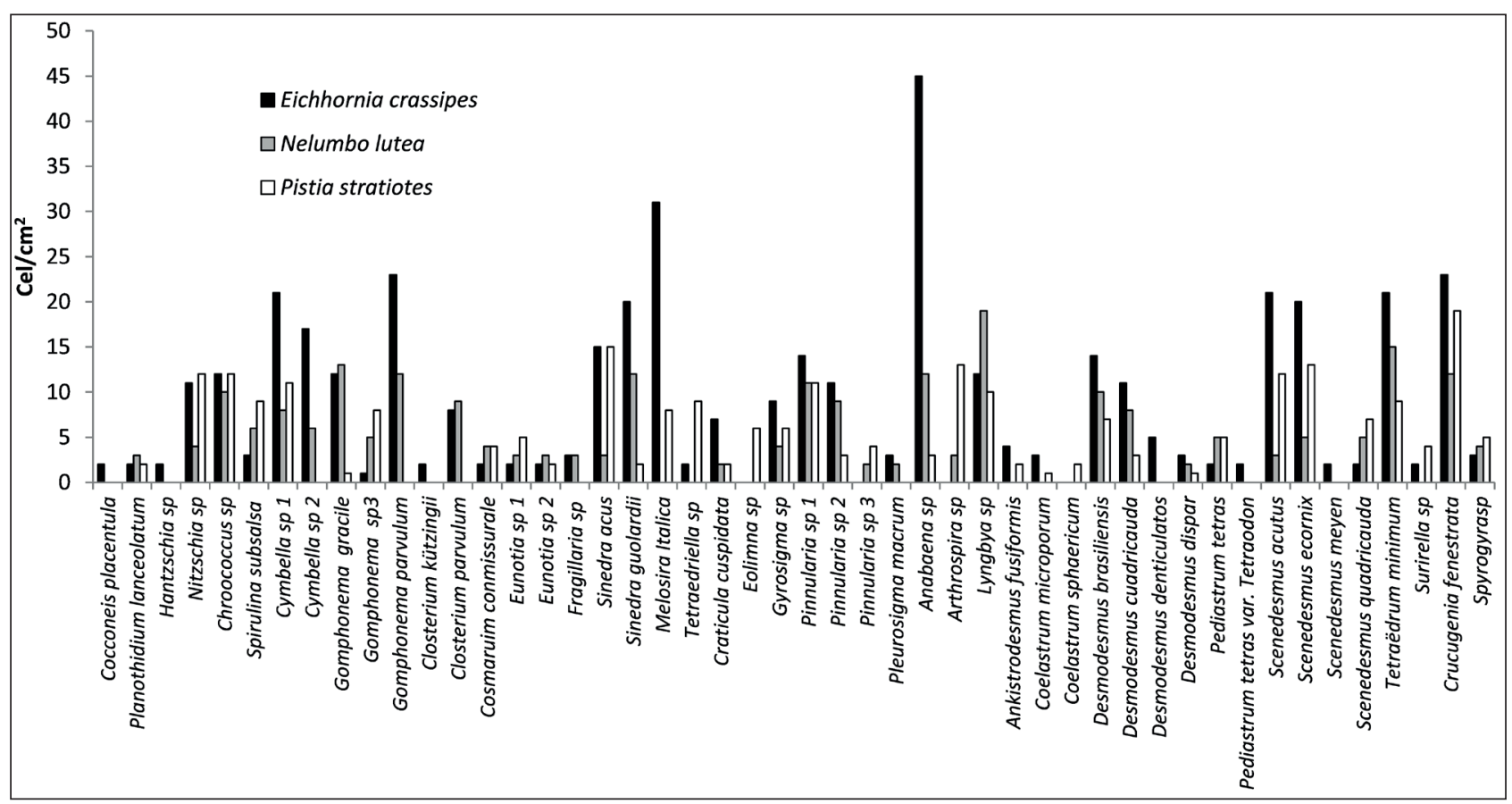

Figura 3. Abundancia de especies ficoperifíticas asociadas a macrófitas en la ciénaga Cerro de San Antonio.

Los índices ecológicos analizados para el ficoperifiton asociado a raíces de macrófitas, mostraron poca diferencia en cuanto a la diversidad de especies en cada macrófita, de esta manera $N$. lutea presentó un valor de diversidad de 3,32 bit/ind, superior a E. crassipes y P. stratiotes que presentaron valores de 3,28 bit/ind y 3,24 bit/ind respectivamente.

En lo que respecta a la riqueza, E. crassipes presentó el mayor valor con 41 especies, seguido por $N$. lutea y $P$. stratiotes quienes presentaron 33 especies cada una. Por otra parte, el índice de dominancia de Simpson, mostró valores de 0,95 para las tres especies de macrófitas (Tabla 1).

Tabla 1. Índices ecológicos del ficoperifiton asociado a raíces macrófitas en la Ciénaga Cerro de San Antonio.

\begin{tabular}{lrrr}
\hline & E. crassipes & $\begin{array}{r}\text { Nelumbo } \\
\text { lutea }\end{array}$ & $\begin{array}{r}\text { Pistia } \\
\text { stratiotes }\end{array}$ \\
\hline Especies & 44 & 35 & 37 \\
Individuos & 432 & 237 & 248 \\
Dominancia D & 0,04399 & 0,04018 & 0,03928 \\
Simpson 1-D & 0,956 & 0,9598 & 0,9607 \\
Shannon H & 3,369 & 3,363 & 3,381 \\
\hline
\end{tabular}

En la figura 4, el análisis de componentes principales (ACP), se relacionan las especies de microalgas perifíticas con las especies de macrófitas dominantes de la zona, (con varianza del primer componente de 77,61 $\%$; del segundo componente de $14,86 \%$ y del tercer componente con 7,51 \%). Se encontraron diferencias en cuanto a la composición y la abundancia de especies. Las macrófitas Pistia stratiotes y Nelumbo lutea, fueron similares en cuanto a la composición de ficoperifiton, lo cual se relacionó con la presencia de Desmodesmus brasiliensis, Nitzschia sp., Synedra acus, Chroococcus sp., Pinnularia sp. (Figura 4). Mientras E. crassipes mostró diferencias marcadas, dadas por las especies Synedra guolardii, Melosira itálica, Gomphonema parvulum y Cymbella sp2. (Figura 4).

El análisis de Cluster mostró el proceso de ordenación de las abundancias de las especies ficoperifíticas con las macrófitas estudiadas, el coeficiente de correlación cofenético fue de 0,85 (Figura 5), este valor fue alto e indica que las distancias en el dendrograma reflejan eficientemente las distancias evaluadas (verdaderas), entre todos datos que se clasificaron. El ordenamiento generó tres subgrupos claramente diferenciados, cada grupo correspondió a una macrófita, así, el primer subgrupo (E. crassipes), fue el de mayor 


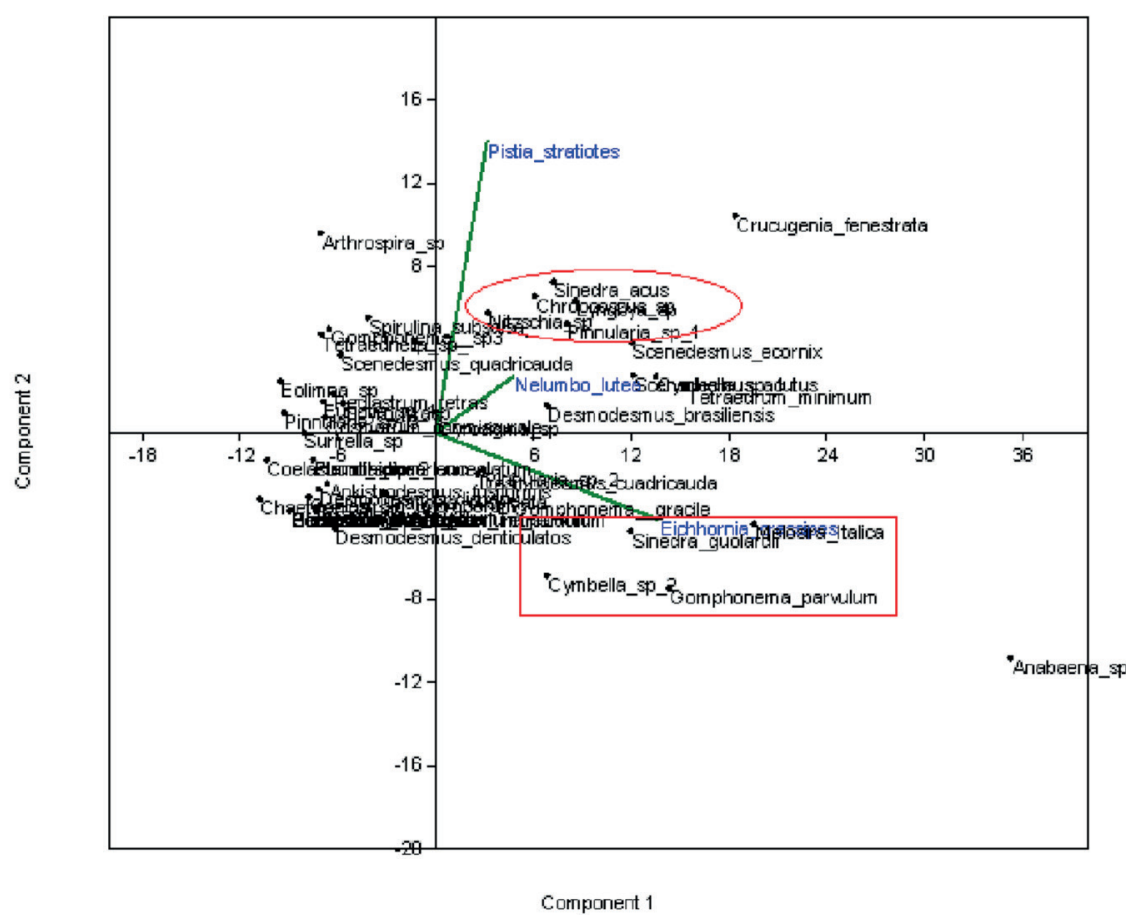

Figura 4. Relación generada por el Análisis de Componentes Principales (ACP), entre las especies de ficoperifíticas asociadas a raíces de E. crassipes, Nelumbo lutea y Pistia stratiotes presentes en la Ciénaga Cerro de San Antonio.

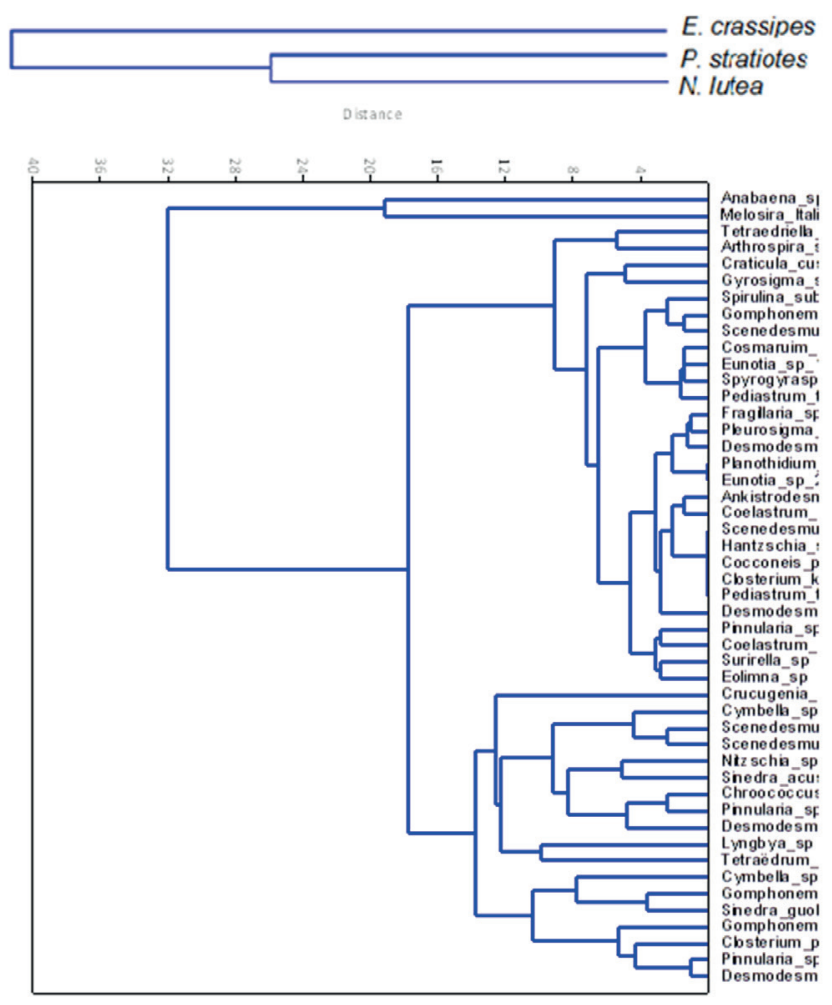

Figura 5. Análisis de agrupamiento (Clúster) de las macrófitas estudiadas y las abundancias ficoperifíticas, con base en el índice de disimilitud (Euclidea). Coeficiente de correlación cofenético 0,85 . disimilitud, la diferencia estuvo dada en las altas abundancias de las especies Anabaena sp. y Melosira italica. El segundo y tercer subgrupos (P. stratiotes y N. lutea respectivamente), presentaron mayor afinidad con relación a la composición de especies ficoperifíticas, sin embargo, se diferencian en las abundancias relativas por especie, por lo que se les puede considerar grupos próximos, o con composición de algas muy similar.

\section{DISCUSIÓN}

En esta investigación se registraron 48 especies ficoperifíticas, cinco más que las encontradas por Montoya y Aguirre (2008), en el complejo cenagoso de Ayapel (Córdoba), en donde evaluaron el ficoperifiton asociado a seis macrófitas, a pesar de que la ciénaga Cerro de San Antonio presentó menor cantidad de especies de macrófitas, se evidenció una alta diversidad de ficoperifíton asociado a éstas. En términos generales la densidad total de algas perifíticas fue baja, esto sugiere una alta carga de materia orgánica y sedimentos (observada al limpiar las raíces), producto de la entrada de agua del caño Cerro de San Antonio; por otro lado, otros factores como la herbivoría, la competencia con el fitoplancton y la competencia inter e intraespecifica, también pudieron contribuir a los resultados encontrados, ya que está ampliamente demostrado que éstos, son los principales factores bióticos que regulan el crecimiento de algas del perifiton (Oliveira-Martins, 2006).

La especie E. crassipes, presentó mayor riqueza y densidad algal, valores atribuidos a que la estructura de la raíz de esta macrófita, es un rizoma de mayor envergadura y más 
ramificado que en $P$. stratiotes, a su vez $P$. stratiotes fue ligeramente superior a $N$. lutea en abundancia y riqueza de especies. Mientras que las raíces de $N$. lutea permanecen arraigadas al sustrato, lo que dificulta la fijación algal; $P$. stratiotes y E. crassipes presentan raíces, en la mayoría de los casos, flotantes en la columna de agua, lo cual puede facilitar la colonización y adherencia de algas con o sin estructuras de fijación. Montoya y Aguirre (2008), registraron para Ayapel un comportamiento similar, dos especies del género Eichhornia presentan los mayores valores de densidad ficoperifítica, en donde al igual que en esta investigación el promedio de densidad algal se incrementó de acuerdo a la complejidad estructural de la raíz sustrato (Montoya y Aguirre, 2008).

Por otro lado, E. crassipes y P. stratiotes, fueron los sustratos por los que algunas especies ficoperifíticas mostraron algún tipo de preferencia. Las especies $C$. kützingii, Cocconeis placentula, Hantzschia sp., P. tetras y $S$. meyen, presentes únicamente en las raíces de E. crassipes y las algas Eolimna sp. y C. sphaericum presentes exclusivamente en raíces de P. stratiotes, presentaron bajas densidades, por lo que no se puede determinar si existe algún tipo de agremiación microalga-macrófita. A su vez, algunas de estas especies son de hábito planctónico por lo que su presencia en el sustrato puede ser accidental y deberse a la polimixis del sistema, como lo documentaron Montoya y Aguirre (2013a), en la ciénaga de Ayapel.

Las diatomeas fueron la clase dominante, esta dominancia se debe a que este grupo presenta gran flexibilidad ecológica lo que les permite colonizar diversos nichos y alcanzar una amplia diversificación, estando presentes en ambientes con diferentes niveles tróficos (Margalef, 1983). Algunas diatomeas tienen estructuras especializadas para fijarse al sustrato, como pedúnculos mucilaginosos (Ej. Gomphonema), secreción y producción de una matriz mucilaginosa (Ej. Cymbella, Frustulia y Navicula) y formas con ramas o pedúnculos que permiten la formación de colonias fijas al sustrato como en Eunotia y Fragillaria (Montoya y Aguirre, 2013a). Los altos valores de abundancia y riqueza de especies de estos géneros, podrían estar relacionados con la presencia de estructuras especializadas que les permiten competir con ventaja, sobre otras especies 80 en condiciones ambientales estresantes, impuestas por la velocidad de la corriente y las descargas de contaminantes (Rodríguez y Bicudo, 2001).

Se encontraron géneros de algas verdes (clorofíceas), de habito plantónico presentes en el ensamble ficoperifítico
(Pediastrum, Desmodesmus, Spyrogira, Cosmarium, entre otros), comunes de aguas mesotróficas a oligotróficas (Ramírez, 2000). Su presencia se debe a lo poco profundo del ecosistema y a la acción del viento que mezcla continuamente el agua, lo cual pudo arrastrar a estos organismos plantónicos a la matriz perifítica en donde son embebidos con facilidad. En estudios de perifiton de ambientes lénticos, embalses y sistemas acuáticos como humedales, es común encontrar que las diatomeas predominen en ensambles perifíticos; sin embargo, en las ciénagas la dominancia de las diatomeas se iguala a las clorofíceas e incluso son reemplazadas en el dominio del ensamble, como ocurre en ecosistemas en el Chocó (Montoya-Moreno y Aguirre, 2013b). De acuerdo con los datos registrados en la Ciénaga Cerro de San Antonio, las diatomeas comparten dominancia con las clorofíceas, aunque por las altas densidades registradas para algunas especies de clorofíceas, la tendencia es que éstas se vean favorecidas y dominen por completo la comunidad.

La especie Ankistrodesmus fusiformis (Figura 6B), es de hábitat planctónico y cosmopolita (Ramos et al., 2012) y está presente en lagos dulceacuícolas o asociado a vegetación del litoral. El registro de esta especie representa el primero para las ciénagas del departamento del Magdalena. Como la mayoría de especies registrada, A. fusiformis prefiere aguas poco ácidas y no contaminadas y son sensibles a los herbicidas (Echenique et al., 2004).

El género Closterium (Figura 6A) es cosmopolita, está presente en el perifiton de lagos y lagunas oligotróficas ácidas; rara vez en aguas alcalinas y ambientes eutróficos (Guiry, 2015). Las especies de este género son particularmente resistentes a los desechos ricos en cromo y cuando su densidad es alta, pueden conferirle olor a pepino al agua (Ramírez, 2000).

Dentro de las cianofíceas, la especie Anabaena sp. (Figura 6D), presentó valores significativos de abundancia, en las tres macrófitas evaluadas; resultado esperado debido a que es una especie común de aguas estancadas en donde se encuentra formando parte del plancton (De la Lanza et al., 2000). Esta Cyanophyceae, es capaz de detectar de forma general cualquier tipo de elemento ambiental perjudicial que le suponga una destrucción en su actividad metabólica, el cual se traduce en una disminución de la emisión de luz del organismo, lo que se utiliza como indicador de toxicidad ambiental (De la Lanza et al., 2000). Dicha propiedad luminiscente, se emplea de forma rutinaria para la medida rápida de toxicidad de muestras ambientales de 
toda índole (aguas fluviales, efluentes de depuradoras, aguas marinas, subterráneas, suelos, etc., De la Lanza et al., 2000).

La cianofícea Spirulina subsalsa (Figura 6C), es cosmopolita y de amplia distribución (Guiry, 2015). Habitualmente se encuentra de manera solitaria, mezclada con otras algas, en diversos ambientes, que abarcan desde aguas dulces o salinas hasta humedales costeros, en los que vive de manera planctónica y bentónica. No suele formar poblaciones abundantes. Fue registrada en 1996 en la Ciénaga Grande de Santa Marta (CGSM), sin embargo no se proporcionan registros fotográficos (Vidal, 2010).

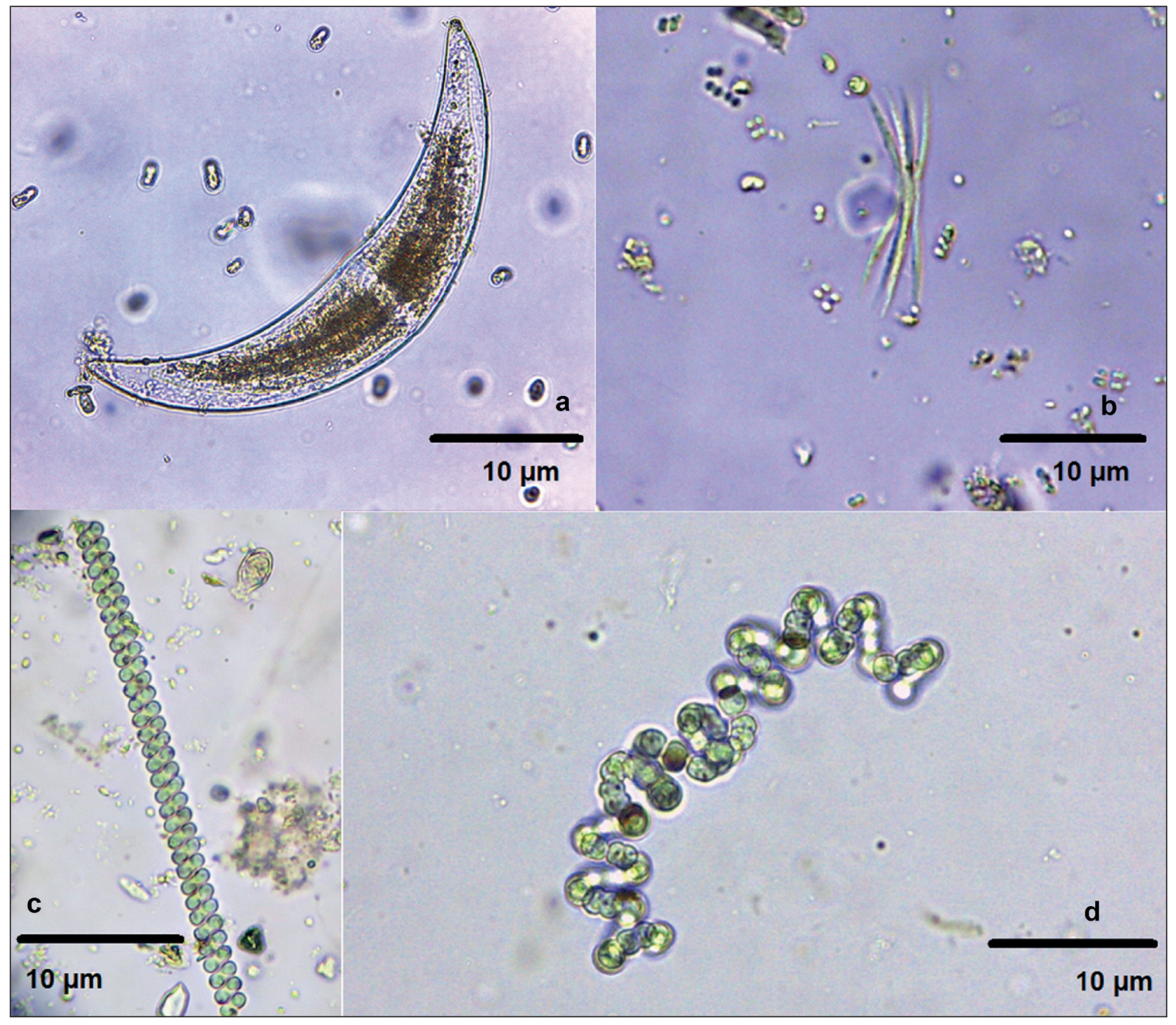

Figura 6. Especies asociadas a raíces de macrófitas en la ciénaga Cerro de San Antonio. A: Closterium parvulum; B: Ankistrodesmus fusiformis; C: Spirulina subsalsa, D: Anabaena sp.

\section{CONCLUSIONES}

La caracterización del ensamble ficoperifítico, dio como resultado la co-dominancia de las diatomeas y clorofíceas, en todos los sustratos evaluados, por lo general las diatomeas predominan en este tipo de ambientes ya que presentan estructuras y adaptaciones para la fijación al sustrato (pedúnculos, ganchos, sustancias mucilaginosas), sin embargo la presencia en altas densidades de clorofíceas, puede indicar que esta ciénaga recibe una carga orgánica elevada (evidenciada al limpiar las raíces) y una penetración lumínica permanente a lo largo de todo el año que favorece su crecimiento. 
En el ensamble ficoperifítico estudiado son más notorias las diferencias en cuanto a la densidad de algas de cada sustrato particular. Sólo E. crassipes y $P$. stratiotes presentaron especies exclusivas, sin embargo, el muestreo realizado no es suficientemente representativo para afirmar que la selectividad por el sustrato de algunas especies de algas, se deba a algún tipo de agremiación microalga-macrófita.

\section{AGRADECIMIENTOS}

Este estudio fue realizado en el marco del proyecto INVESTIGACIÓN PARA LA CARACTERIZACIÓN, ZONIFICACIÓN, ORDENAMIENTO, RESTAURACIÓN Y MANEJO DE CIÉNAGAS DEPARTAMENTO DEL MAGDALENA, CARIBE. Código BPIN: 2013000100017, financiado bajo el Convenio de cooperación 096/2013 entre la Fundación para la Participación; Capacitación y la Investigación Social "FUPARCIS" y la Gobernación del Magdalena.

\section{BIBLIOGRAFÍA}

Bicudo, E.M. y Menezes, M. 2006 Gêneros de algas de águas continentais. do Brasil (Chave de identificação e descrições). 2 Ed. RiMa Editora, São Carlos, Brasil.

Bourrelly, P. 1970. Les algues d'eau douce. N. Boubée y Cie. Paris.

Bourrelly, P. 1972. Les algues d'eau douce. N. Boubée y Cie. Paris.

Bourrelly, P. 1981. Les algues d'eau douce. N. Boubée y Cie. Paris.

CEN/TC 230. 2002. Water quality-Guidance standard for the routine sampling and pretreatment of benthic diatoms from rivers. European Standard. Standard references.

Echenique, R.O., Núñez-Avellaneda, M. y Duque, S.R. 2004. Chlorococcales de la Amazonia colombiana I: Chlorellaceae y Scenedesmaceae. Revista Caldasia 26(1): 37-51.

De la Lanza G.S., Pulido, H. y Carvajal, J.L.P. 2000. 82 Organismos indicadores de la calidad del agua y de la contaminación (bioindicadores). Plaza y Valdez / Comisión Nacional del Agua, SEMARNAP/Instituto de Biología, UNAM, México, D.F.
Flynn, N.J., Snooka, D.L., Wadea, A.J. y Jarvieb, H.P. 2002. Macrophyte and periphyton dynamics in a UK Cretaceous chalk stream: the River Kennet, a tributary of the Thames. Science of the Total Environment 282-283: 143-57.

Guiry, M.D. 2015. AlgaeBase. World-wide electronic publication, National University of Ireland, Galway. http:// www.algaebase.org. Consultado: 26 de Noviembre 2015.

Hauer, F.R. y Lamberti, G.A. (Eds). 2007. Methods in stream ecology. Academic Press. San Diego.

Hernández-Atilano, E.N., Aguirre, J. y Palacio, J.A. 2005. Variación espacio-temporal de la estructura de la comunidad de algas perifíticas en la microcuenca de la quebrada la vega, municipio de San Roque (Antioquia), Colombia. Actualidades Biológicas 27(82): 67-77.

Krammer, K. y Lange-Bertalot, H. 1986. Bacillariophyceae. In: Verfasser, Herausgeber y Mollenhauer. Süsswasserflora von Mittleuropa. Band 1, Teil. Naviculaceae. Jena: Gustav Fisher Verlag.

Krammer, K. y Lange-Bertalot, H. 1988. 2/2 Baclllariophyceae. 2. Teil: Bacillariaceae, Epithemiaceae, Surirellaceae. In: Ettl, H., Gerloff, J., Heyning, H. y Mollenhauer, D. (Eds). Süßwasserflora von Mitteleuropa. Stuttgart. Gustav Fischer Verlag.

Krammer, K. y Lange-Bertalot, H. 1991. 2/3 BacIllariophyceae. 2. Teil: Centrales, Fragilariaceae, Eunotiaceae. In: Ettl, H., Gerloff, J., Heyning, H. y Mollenhauer, D. (Eds). Süßwasserflora von Mitteleuropa. Stuttgart, Gustav Fischer Verlag.

Lange-Bertalot, H. 2001. Diatoms of Europe. Diatoms of the European Inland Waters and Comparable Habitats. Navicula sensu stricto 10 Genera separated from Navicula sensu lato. Frustulia. Ruggell: Koeltz Scientific Books.

Margalef, R. 1983. Limnología. 1st edition. Omega. Barcelona.

McCormick, P.V. y Stevenson, R.J. 1998. Periphyton as a tool for ecological assessment and management in the Florida Everglades. Journal Phycology 34: 726-733.

Montoya-Moreno, Y. y Aguirre-Ramírez, N. 2008. Asociación de algas perifíticas en raíces de macrófitas en una ciénaga tropical Colombiana. Hidrobiológica 18(3): 189-198. 
Montoya-Moreno, Y. y Aguirre, N. 2009. Estado del arte de la limnología de lagos de planos inundables (ciénagas) en Colombia. Revista Gestión y Ambiente 12(3): 85-106.

Montoya-Moreno, Y. y Aguirre, N. 2013a. Dinámica del ensamblaje algal epifítico en el sistema de planos inundables de Ayapel a través del pulso de inundación. Revista U.D.C.A Actualidad y Divulgación Científica 16(2): 491-500.

Montoya-Moreno, Y. y Aguirre, N. 2013b. Estado de arte del conocimiento sobre perifíton en Colombia. Revista Gestión y Ambiente 16(3): 91-117.

Montoya-Moreno, Y. y Ramírez, J.J. 2007. Variación estructural de la comunidad perifítica colonizadora de sustratos artificiales en la zona de ritral del río Medellín, Colombia. Revista de Biología Tropical 55(2): 585-593.

Oliveira-Martins, F. 2006. Respostas ecofisiologicas da comunidade perifitica in situ a diferentes condicoes ambientais no Rio Santa da Vitoria. Es. Tesis doctoral. Universidade Federal Do Espiritu Santo.

Ramírez, J. 2000. Fitoplancton de agua dulce: aspectos ecológicos, taxonómicos y sanitarios. Tesis de Maestría, Universidad de Antioquia, Medellín, Colombia.

Ramos, G.J.P., Bicudo, C.E. de M., Góes-Neto, A. y Moura, C.W. do N. 2012. Monoraphidium and Ankistrodesmus (Chlorophyceae, Chlorophyta) from Pantanal dos
Marimbus, Chapada Diamantina, Bahia State, Brazil. Hoehnea 39(3): 421-434.

Rivera, C. y Donato, J. 2008. Influencia de las variaciones hidrológicas y químicas sobre la diversidad de diatomeas bénticas. En: Donato, J., Editor. Ecología de un rio de montaña de los Andes colombianos (Rio Tota, BoyacáColombia). Universidad Nacional de Colombia, Bogotá D.C.

Rodríguez, L. y Bicudo, D. 2001. Similarity among periphyton algal communities in a lentic-lotic gradient of the upper Paraná River floodplain, Brazil. Revista Brasilera de Botánica 3: 235-48.

Sand-Jensen, K. 1983. Physical and chemical parameters regulating growth of Periphytic communities. En: Wetzel, R.G. Editor. Periphyton of Freshwater Ecosystems. Boston.

Sierra, O. y Ramírez, J.J. 2000. Variación espacio-temporal de biopelículas en la represa La Fe, el Retiro, Antioquia (Colombia). Actualidades Biológicas 22(73): 153-168.

Vidal, L.A. 2010. Manual de fitoplancton hallado en la Ciénaga grande de Santa Marta y cuerpos de agua aledaños. Fundación universitaria de Bogotá Jorge Tadeo Lozano.

Wilby, R., Cranston, L.E. y Darby, E.J. 1998. Factors governing macrophyte status in Hampshire chalk streams: implications for catchment management. Water and Environment Journal 12: 179-87.

Fecha de recepción: 31/08/2015 Fecha de aceptación: 23/11/2015

Para citar este artículo: Osorio-Ávila, F. y G. Manjarres-García. 2015. Ficoperifiton asociado a macrófitas en la ciénaga Cerro de San Antonio, Magdalena-Colombia. Intropica Vol. 10: 74 - 83 